\title{
Propriedades físicas do solo e produtividade de grãos de milho cultivo após espécies
}

\section{de cobertura}

\author{
Soil physical properties and maize grain yield after cover Crops \\ Propiedades físicas del suelo y rendimiento de grano de maíz después de cultivos de cobertura
}

Recebido: 23/11/2021 | Revisado: 29/11/2021 | Aceito: 30/11/2021 | Publicado: 11/12/2021

Mayra Beatriz Semiano Castro

ORCID: https://orcid.org/0000-0002-2818-6623

Universidade Estadual do Oeste do Paraná, Brasil

E-mail: mayrabscastro@gmail.com

Deonir Secco

ORCID: https://orcid.org/0000-0002-3042-159X

Universidade Estadual do Oeste do Paraná, Brasil

E-mail: deonir.secco@ unioeste.br

Araceli Ciotti de Marins

ORCID: https://orcid.org/0000-0001-8932-7015

Universidade Tecnológica Federal do Paraná, Brasil E-mail: araceli@utfpr.edu.br

Doglas Bassegio

ORCID: https://orcid.org/0000-0001-6628-8594

Universidade Estadual do Oeste do Paraná, Brasil E-mail: doglas.bassegio@unioeste.br

Samuel Nelson Melegari de Souza

ORCID: https://orcid.org/0000-0003-2455-7279

Universidade Estadual do Oeste do Paraná, Brasil

E-mail: samuel.souza@unioeste.br

Luiz Antonio Zanão Junior

ORCID: https://orcid.org/0000-0003-4159-1380

Instituto de Desenvolvimento Rural do Paraná, Brasil

E-mail: samuel.souza@unioeste.br

\begin{abstract}
Resumo
Estudos envolvendo plantas de cobertura em áreas manejadas sob sistema plantio direto são de grande relevância cientifica para buscar a sustentabilidade dos cultivos agrícolas. O presente trabalho teve por objetivo avaliar o impacto do uso de plantas de cobertura na estrutura de um Latossolo argiloso sob sistema plantio direto e o reflexo na produtividade do milho. Os tratamentos consistiram na utilização de seis espécies de cobertura: milheto (Pennisetum glaucum), guandu-forrageiro-anão (Cajanus cajan), crotalária (Crotalaria juncea), feijão-guandu (Cajanus cajan); crotalaria spectabilis (Crotalaria spectabilis Roth); mucuna preta (Stizolobium aterrimum) e do sistema plantio direto tradicional (testemunha). O delineamento experimental utilizado foi inteiramente casualizado com 4 repetições. Os parâmetros físicos do solo avaliados foram a densidade, o espaço poroso e a condutividade hidráulica do solo. Para isso, foram coletadas amostras indeformadas de solo nas camadas de 0,00-0,10 m, 0,10-0,20 m e 0,20-0,30 m. Após dessecação das espécies de cobertura, em plena floração, implantou-se a cultura do milho. O rendimento de grãos de milho foi determinado a partir de 3 repetições de $13,5 \mathrm{~m}^{2}$ de área cada. A umidade dos grãos foi corrigida para $13 \%$ para a determinação da produtividade. As espécies de cobertura de verão que apresentaram maior eficiência na reestruturação do solo foram a crotalária spectabilis e a mucuna preta. Quanto ao rendimento de grãos de milho não houve diferença significativa entre os tratamentos, evidenciando as boas condições estruturais do solo da área experimental.
\end{abstract}

Palavras-chave: Parâmetros físicos do solo; Estrutura do solo; Rendimento de grãos do milho.

\begin{abstract}
Studies involving cover crops in areas managed under no-tillage system are of great scientific relevance to seek the sustainability of agricultural crops. This study aimed to evaluate the impact of the use of cover crops on the structure of a clayey Oxisol under no-tillage system and its effect on corn yield. The treatments consisted of the use of six cover species: millet (Pennisetum glaucum), dwarf pigeon pea (Cajanus cajan), sunn hemp (Crotalaria juncea), pigeon pea (Cajanus cajan); crotalaria spectabilis (Crotalaria spectabilis Roth); black velvet bean (Stizolobium aterrimum) and the traditional no-tillage system (control). The experimental design used was completely randomized with 4 replications. The soil physical parameters evaluated were density, pore space and soil hydraulic conductivity. For this
\end{abstract}


purpose, undisturbed soil samples were collected in the $0.00-0.10 \mathrm{~m}, 0.10-0.20 \mathrm{~m}$ and $0.20-0.30 \mathrm{~m}$ layers. After desiccation of the cover species, in full bloom, the corn crop was implemented. The corn grain yield was determined from 3 replicates of $13.5 \mathrm{~m}^{2}$ of area each. Grain moisture was corrected to $13 \%$ to determine yield. The summer cover species that showed greater efficiency in soil restructuring were crotalaria spectabilis and black velvet bean. As for corn grain yield, there was no significant difference between treatments, evidencing the good structural conditions of the soil in the experimental area.

Keywords: Soil physical parameters; Soil structure; Corn grain yield.

\section{Resumen}

Los estudios que involucran cultivos de cobertura en áreas manejadas bajo el sistema de labranza cero son de gran relevancia científica para buscar la sustentabilidad de los cultivos agrícolas. Este estudio tuvo como objetivo evaluar el impacto del uso de cultivos de cobertura en la estructura de un Oxisol arcilloso bajo un sistema de labranza cero y su efecto en el rendimiento del maíz. Los tratamientos consistieron en el uso de seis especies de cobertura: mijo (Pennisetum glaucum), gandul enano (Cajanus cajan), cáñamo (Crotalaria juncea), gandul (Cajanus cajan); Crotalaria spectabilis (Crotalaria spectabilis Roth); frijol terciopelo negro (Stizolobium aterrimum) y el sistema tradicional de labranza cero (control). El diseño experimental utilizado fue completamente al azar con 4 repeticiones. Los parámetros físicos del suelo evaluados fueron densidad, espacio poroso y conductividad hidráulica del suelo. Para este propósito, se recolectaron muestras de suelo inalterado en las capas de 0.00-0.10 m, 0.10-0.20 my 0.20-0.30 m. Luego de la desecación de las especies de cobertura, en plena floración, se implementó el cultivo de maíz. El rendimiento de grano de maíz se determinó a partir de 3 repeticiones de 13,5 $\mathrm{m}^{2}$ de área cada una. La humedad del grano se corrigió al 13\% para determinar el rendimiento. Las especies de cobertura de verano que mostraron mayor eficiencia en la reestructuración del suelo fueron el cáñamo spectabilis y el frijol terciopelo negro. En cuanto al rendimiento de grano de maíz, no hubo diferencia significativa entre tratamientos, evidenciando las buenas condiciones estructurales del suelo en el área experimental.

Palabras clave: Parámetros físicos del suelo; Estructura del suelo; Rendimiento de grano de maíz.

\section{Introdução}

O Brasil foi o terceiro maior produtor mundial de milho com uma safra anual de 91.08 milhões de toneladas. O Paraná foi responsável por $16,05 \%$ da produtividade nacional com média de $5944 \mathrm{~kg} \mathrm{ha}^{-1}$ (Conab, 2018). Para manter a sustentabilidade do ambiente produtivo, são necessárias técnicas que mantenham adequada as propriedades físicas, químicas, biológicas do solo e juntamente minimizem impactos sob os recursos naturais (Portugual et al., 2017). Nesse intuito, o uso de plantas de cobertura é fundamental para a sustentabilidade dos sistemas de cultivo (Carvalho et al., 2009), as quais possibilitam a melhora da estrutura do solo, amenizando a compactação, contribuindo para o incremento da matéria orgânica do solo.

Quando o sistema de manejo é realizado sem rotação de culturas e com tráfego intensivo de máquinas e equipamentos, sem levar em conta as condições de umidade do solo, causa a compactação subsuperficial (Tokura et al., 2018, Spliethoff et al., 2020; Bareta Junior et al., 2021; Tokura et al., 2021). Desta forma, as práticas de manejo do solo, são atividades que mais influências no comportamento físico, uma vez que, atua diretamente sobre sua estrutura e causa modificações na porosidade e densidade, o que afeta a retenção de água (Warrick \& Nielsen, 1980).

Assim, a utilização de plantas "recuperadoras" de estrutura é uma forma de diminuir a resistência à penetração e a compactação em Latossolo argiloso manejados sob sistema plantio direto (Sulzbach et al., 2017), pois os resíduos culturais podem trazer, além dos benefícios químicos, melhorias na qualidade física do solo (Zin et al., 2019). Algumas espécies apresentam crescimento radicular capaz de se desenvolver em solos com alta resistência à penetração, além disso realizam a ciclagem de nutrientes (Tokura et al., 2018).

Assim, estudos sobre espécies de cobertura são necessários para averiguar qual é o seu impacto na melhoria nos atributos do solo. Além disso, elas são capazes de produzir diferentes quantidades de resíduos vegetais (parte aérea e raízes), que em decomposição alteram os atributos físicos e químicos do solo, refletindo no desenvolvimento e na produtividade da cultura sucessora (Tokura et al., 2018).

Este trabalho tem por objetivo, avaliar a eficácia de espécies de cobertura de verão na recuperação da estrutura de um Latossolo argiloso e seus reflexos no rendimento de grãos na cultura do milho (Zea mays). 


\section{Metodologia}

O experimento foi realizado na estação experimental de Santa Tereza do Oeste/PR do Instituto Agronômico do Paraná-IAPAR. A área experimental foi constituída por sete parcelas de 20x16 metros, em delineamento experimental inteiramente casualizado. Os tratamentos constaram de seis parcelas em sistema plantio direto cultivadas com espécies de cobertura vegetal de verão: milheto (MI), crotalária juncea (CJ), guandú-anão (GA), crotalária spectabilis (CS), feijão guandu (FG) e mucuna preta (MP), além do sistema plantio direto tradicional (SPDT-Testemunha).

A área experimental vem sendo conduzida com cultivos agrícolas em sistema plantio direto há pelo menos dezoito anos. Nesta área foram aplicados os tratamentos e realizadas as avaliações propostas neste estudo. A Tabela 1 apresenta a caracterização química inicial do solo do local.

Tabela 1. Caracterização química inicial do solo antes da implantação do experimento na camada de 0-0,20 m no ano de 2010 .

\begin{tabular}{|c|c|c|c|c|c|c|c|c|c|c|c|}
\hline $\mathrm{P}$ & $\mathrm{C}$ & $\mathrm{pH}$ & $\mathrm{Al}^{3+}$ & $\mathrm{H}^{+}+\mathrm{Al}^{3+}$ & $\mathrm{Ca}^{2+}$ & $\mathrm{Mg}^{2+}$ & $\mathrm{K}^{+}$ & SB & CTC & V & $\mathrm{Al}^{*}$ \\
\hline $\begin{array}{c}\mathrm{mg} \\
\mathrm{dm}^{-3}\end{array}$ & $\mathrm{~g} \mathrm{dm}^{-3}$ & $\mathrm{CaCl}_{2}$ & & ---- & -------- & $---\mathrm{cmo}$ & $-3-\cdots$ & & & ----- & \\
\hline 23,6 & 32,72 & 4,70 & 0,32 & 9,00 & 4,20 & 3,00 & 0,44 & 7,64 & 16,64 & 45,91 & 4,02 \\
\hline
\end{tabular}

Fonte: Autores.

O solo do local é classificado como Latossolo Vermelho Distroférrico típico (LVdf) de textura argilosa (EMBRAPA, 2018). Os resultados da análise granulométrica das três camadas de solo realizada anteriormente a implantação das plantas de cobertura se encontram na Tabela 2.

Tabela 2. Granulometria ${ }^{1}$ do solo da área experimental nas três camadas avaliadas. ${ }^{1}$ Método da pipeta, conforme USDA-Soil Conservation Service. Soil Survey Investigations Report n.1. Whashington, p. 63, 1972.

\begin{tabular}{cccc}
\hline \multirow{2}{*}{ Camada } & \multicolumn{3}{c}{ Frações Granulométricas } \\
\cline { 2 - 4 }$(\mathrm{m})$ & Areia & Argila & Silte \\
\hline $0,00-0,10$ & 44,9 & 561,1 & 394,1 \\
$0,10-0,20$ & 38,7 & 641,9 & 319,4 \\
$0,20-0,30$ & 24,7 & 706,2 & 269,1 \\
\hline Média & 36,1 & 636,4 & 327,5 \\
\hline
\end{tabular}

Fonte: Autores.

Para a determinação da densidade, macroporosidade e condutividade hidráulica saturada do solo, foram coletadas amostras indeformadas em três camadas: 0-0,10; 0,10-0,20 e 0,20-0,30 m, sempre considerando a profundidade intermediária da camada visada, utilizando cilindros de dimensões $0,05 \mathrm{~m}$ de altura e 0,05 $\mathrm{m}$ de diâmetro. Foram coletadas amostras em duplicata em cada camada, em três pontos aleatórios de cada unidade experimental, a fim de obter amostras representativas, considerando a grande dispersão dos atributos físicos e prevenindo perda de amostras durante a realização dos procedimentos laboratoriais.

Para a avaliação da densidade do solo e macroporosidade, foi utilizada uma coluna de areia que permite a extração seletiva de água a tensões pré-estabelecidas Reinert e Reichert (2006). Posteriormente as amostras foram pesadas e saturadas novamente por 24 horas para a determinação da condutividade hidráulica saturada em permeâmetro de carga constante. Os cálculos para obtenção da condutividade hidráulica do solo foram realizados conforme metodologia preconizada por EMBRAPA (1997). 
A semeadura do milho foi realizada no dia 08/03/2018 com o híbrido 90XB06 Bt, sendo que as sementes foram tratadas com Vitavax - Thiram 0,3 L/100 kg de sementes. Na semeadura o espaçamento foi de 0,90 m entre linhas, perfazendo um total de 6 sementes por metro linear. Sem adubação de base, com adubação nitrogenada no dia 23/03/2018 com $200 \mathrm{~kg}$ ha ${ }^{1}$ de uréia e adubação com potássio no dia 02/04/2018, com $100 \mathrm{~kg}$ de cloreto de potássio ha ${ }^{-1}$.

A colheita do milho para determinar a produtividade foi realizada manualmente nos dias 28 e 29/08/18, onde se avaliou 3 linhas de 5 metros com 3 repetições (área de 13,5 $\mathrm{m}^{2}$ ) por parcela. As sementes foram limpas, e o peso foi corrigido para $13 \%$ de umidade.

A análise estatística foi obtida por meio da análise da variância e as médias dos tratamentos foram comparadas pelo teste de Tukey a 5 \% de significância no programa SISVAR (Ferreira, 2010).

\section{Resultados e Discussão}

Pode-se observar na Tabela 3, quanto a densidade do solo (DS), que na camada de 0,00-0,10 m, que os tratamentos de CJ e MP foram os que proporcionaram redução nos valores respectivamente de $1,08 \mathrm{Mg} \mathrm{m}^{-3}$ para $0,95 \mathrm{Mg} \mathrm{m}^{-3}$ e de $1,09 \mathrm{Mg} \mathrm{m}^{-}$

${ }^{3}$ para $0,93 \mathrm{Mg} \mathrm{m}^{-3}$. No período anterior à implantação da cultura do milho, o tratamento que apresentou o menor valor foi o de GA $\left(0,93 \mathrm{Mg} \mathrm{m}^{-3}\right)$ não diferindo dos tratamentos de MI $\left(0,98 \mathrm{Mg} \mathrm{m}^{-3}\right), \mathrm{FG}\left(1,01 \mathrm{Mg} \mathrm{m}^{-3}\right), \mathrm{CS}\left(1,00 \mathrm{Mg} \mathrm{m}^{-3}\right)$ e SPDT $(1,05 \mathrm{Mg}$ $\left.\mathrm{m}^{-3}\right)$, diferindo da CJ $\left(1,08 \mathrm{Mg} \mathrm{m}^{-3}\right)$ e MP $\left(1,09 \mathrm{Mg} \mathrm{m}^{-3}\right)$.

Após a colheita da cultura do milho os tratamentos de $\mathrm{GA}\left(0,91 \mathrm{Mg} \mathrm{m}^{-3}\right)$ e $\mathrm{MP}\left(0,93 \mathrm{Mg} \mathrm{m}^{-3}\right)$ foram os que apresentaram o menor valor de DS, não diferindo do MI (1,02 $\left.\mathrm{Mg} \mathrm{m}^{-3}\right), \mathrm{CJ}\left(0,95 \mathrm{Mg} \mathrm{m}^{-3}\right), \mathrm{FG}\left(0,94 \mathrm{Mg} \mathrm{m}^{-3}\right)$ e CS $\left(0,98 \mathrm{Mg} \mathrm{m}^{-}\right.$ $\left.{ }^{3}\right)$, a exceção do SPDT $\left(1,06 \mathrm{Mg} \mathrm{m}^{-3}\right)$. Na Figura 1, observa-se que em ambos os períodos avaliados, os maiores valores de Ds encontram-se na camada de $0,1-0,2 \mathrm{~m}$.

Para a camada de 0,10-0,20 m os tratamentos que tiveram decréscimo nos valores médios da DS foram o CS e o MP, respectivamente de $1,16 \mathrm{Mg} \mathrm{m}^{-3}$ para 1,01 $\mathrm{Mg} \mathrm{m}^{-3}$ e de 1,17 $\mathrm{Mg} \mathrm{m}^{-3}$ para 1,03 $\mathrm{Mg} \mathrm{m}^{-3}$ (Tabela 3). O fato da CS reduzir a DS se justifica devido a mesma apresentar raiz pivotante e profunda capaz de transpor as camadas compactadas, que ao se decomporem, favorecem as culturas subsequentes permitindo o desenvolvimento dos seus sistemas radiculares pelos bioporos produzidos. As melhorias proporcionadas pelas plantas de cobertura também foram observadas por Calegari et al. (1993).

Para a camada de 0,20-0,30m ao analisarmos os dados de DS, vemos que os tratamentos que propiciaram reduções significativas foram o FG, CS e MP com reduções nos valores respectivamente de $1,08 \mathrm{Mg} \mathrm{m}^{-3}$ para $1,00 \mathrm{Mg} \mathrm{m}^{-3}, 1,12 \mathrm{Mg} \mathrm{m}^{-3}$ para $1,04 \mathrm{Mg} \mathrm{m}^{-3}$ e $1,07 \mathrm{Mg} \mathrm{m}^{-3}$ para $0,96 \mathrm{Mg} \mathrm{m}^{-3}$.

Quanto aos efeitos de tratamentos na época antes da implantação da cultura do milho (2018a) verifica-se que a DS do tratamento com MI (0,98 $\left.\mathrm{Mg} \mathrm{m}^{-3}\right)$ foi o que apresentou o menor valor, contudo não diferiu ao FG (1,08 $\left.\mathrm{Mg} \mathrm{m}^{-3}\right)$ e do MP $(1,07$ $\left.\mathrm{Mg} \mathrm{m}^{-3}\right)$, embora tenha apresentado diferença significativa dos tratamentos de $\mathrm{GA}\left(1,11 \mathrm{Mg} \mathrm{m}^{-3}\right), \mathrm{CJ}\left(1,11 \mathrm{Mg} \mathrm{m}^{-3}\right), \mathrm{CS}(1,12$ $\left.\mathrm{Mg} \mathrm{m}^{-3}\right)$ e SPDT $\left(1,15 \mathrm{Mg} \mathrm{m}^{-3}\right)$.

Já na condição após a colheita da cultura do milho (2018d), pode-se observar que o tratamento com menor média da DS foi o MP $\left(0,96 \mathrm{Mg} \mathrm{m}^{-3}\right)$, não diferindo do MI (1,06 $\left.\mathrm{Mg} \mathrm{m}^{-3}\right)$, FG $\left(1,00 \mathrm{Mg} \mathrm{m}^{-3}\right)$ e CS $\left(1,04 \mathrm{Mg} \mathrm{m}^{-3}\right)$, diferindo-se dos tratamentos de GA $\left(1,11 \mathrm{Mg} \mathrm{m}^{-3}\right)$, CJ $\left(1,13 \mathrm{Mg} \mathrm{m}^{-3}\right)$ e SPDT (1,09 $\left.\mathrm{Mg} \mathrm{m}^{-3}\right)$, evidenciando desta forma, que após a senescência das raízes, os bioporos deixados pelas diferentes plantas de cobertura apresentaram efeitos positivos na redução da DS para a cultura sucessora.

Os baixos valores de DS na camada de 0,00-0,10m são explicados devido ao maior acúmulo de matéria orgânica, bem como revolvimento pelos mecanismos da semeadora no momento da semeadura das culturas. Desta forma pode-se observar que a média geral dos tratamentos tem menor valor de densidade nesta camada (Tabela 3, Figura 1). 
Verifica-se que a camada de 0,00-0,10m apresentou valor médio de $1,0 \mathrm{Mg} \mathrm{m}^{-3}$ de densidade e a camada de 0,10$0,20 \mathrm{~m}$ apresenta o maior valor com $1,11 \mathrm{Mg} \mathrm{m}^{-3}$. Estando os valores abaixo dos valores considerados críticos, que segundo Reichert et al. (2009) para solos argilosos é de 1,35 $\mathrm{Mg} \mathrm{m}^{-3}$ ou superior.

Tabela 3. Valores médios da densidade (DS) e condutividade hidráulica do solo saturado (Ksat) do solo nas camadas de 0,00 0,10, 0,10-0,20, 0,20-0,30 m, antes da implantação (2018a) e após a colheita da cultura do milho (2018b) (valores médios de três repetições). Médias de tratamentos seguidas de mesmas letras, minúscula na coluna e maiúscula na linha, não diferem entre si pelo Teste de Tukey a 5\% de significância.

\begin{tabular}{|c|c|c|c|c|}
\hline Tratamentos & \multicolumn{2}{|c|}{$\mathrm{DS}\left(\mathrm{Mg} \mathrm{m}^{-3}\right)$} & \multicolumn{2}{|c|}{ Ksat $\left(\mathrm{mm} \mathrm{h}^{-1}\right)$} \\
\hline \multicolumn{5}{|c|}{ Camada de 0,00-0,10 m } \\
\hline & $2018 \mathrm{a}$ & $2018 d$ & $2018 \mathrm{a}$ & $2018 d$ \\
\hline MI & $0,98 \mathrm{abA}$ & $1,02 \mathrm{abA}$ & $24,07 \mathrm{aA}$ & $51,64 \mathrm{aA}$ \\
\hline GA & $0,93 \mathrm{bA}$ & $0,91 \mathrm{bA}$ & $67,64 \mathrm{aB}$ & $236,19 \mathrm{aA}$ \\
\hline CJ & $1,08 \mathrm{aA}$ & $0,95 \mathrm{abB}$ & $84,26 \mathrm{aA}$ & $52,65 \mathrm{aA}$ \\
\hline FG & $1,01 \mathrm{abA}$ & $0,94 \mathrm{abA}$ & $32,22 \mathrm{aB}$ & $177,30 \mathrm{aA}$ \\
\hline $\mathrm{CS}$ & $1,00 \mathrm{abA}$ & $0,98 \mathrm{abA}$ & $16,24 \mathrm{aB}$ & $185,04 \mathrm{aA}$ \\
\hline MP & $1,09 \mathrm{aA}$ & $0,93 \mathrm{bB}$ & $63,22 \mathrm{aA}$ & $154,40 \mathrm{aA}$ \\
\hline SPDT & $1,05 \mathrm{abA}$ & $1,06 \mathrm{aA}$ & $98,97 \mathrm{aA}$ & $52,78 \mathrm{aA}$ \\
\hline \multicolumn{5}{|c|}{ Camada de 0,10-0,20 m } \\
\hline MI & $1,06 \mathrm{aA}$ & $1,06 \mathrm{aA}$ & $30,50 \mathrm{aA}$ & $27,41 \mathrm{bA}$ \\
\hline GA & $1,17 \mathrm{aA}$ & $1,11 \mathrm{aA}$ & $3,68 \mathrm{bA}$ & $23,29 \mathrm{bA}$ \\
\hline CJ & $1,12 \mathrm{aA}$ & $1,14 \mathrm{aA}$ & $21,22 \mathrm{bA}$ & $38,73 \mathrm{bA}$ \\
\hline FG & $1,14 \mathrm{aA}$ & $1,11 \mathrm{aA}$ & $48,17 \mathrm{bB}$ & $191,45 \mathrm{aA}$ \\
\hline CS & $1,16 \mathrm{aA}$ & $1,01 \mathrm{aB}$ & $14,78 \mathrm{bB}$ & $137,59 \mathrm{abA}$ \\
\hline MP & $1,17 \mathrm{aA}$ & $1,03 \mathrm{aB}$ & $9,69 \mathrm{bA}$ & $76,41 \mathrm{abA}$ \\
\hline SPDT & $1,16 \mathrm{aA}$ & $1,11 \mathrm{aA}$ & $85,48 \mathrm{abA}$ & $40,31 \mathrm{bA}$ \\
\hline \multicolumn{5}{|c|}{ Camada de 0,20-0,30 m } \\
\hline MI & $0,98 \mathrm{bB}$ & $1,06 a b c A$ & $51,43 \mathrm{aA}$ & 72,37abA \\
\hline GA & $1,11 \mathrm{aA}$ & $1,11 \mathrm{abA}$ & $12,56 \mathrm{aA}$ & $10,81 \mathrm{bA}$ \\
\hline CJ & $1,11 \mathrm{aA}$ & $1,13 \mathrm{aA}$ & $23,17 \mathrm{aA}$ & $9,38 \mathrm{bA}$ \\
\hline FG & $1,08 \mathrm{abA}$ & $1,00 \mathrm{bcB}$ & $97,59 \mathrm{aB}$ & $278,90 \mathrm{aA}$ \\
\hline CS & $1,12 \mathrm{aA}$ & $1,04 \mathrm{abcB}$ & $67,59 \mathrm{aA}$ & $205,96 a b A$ \\
\hline MP & $1,07 \mathrm{abA}$ & $0,96 \mathrm{cB}$ & $160,89 \mathrm{aA}$ & $225,44 \mathrm{abA}$ \\
\hline SPDT & $1,15 \mathrm{aA}$ & $1,09 \mathrm{abA}$ & $59,46 \mathrm{aA}$ & $26,87 \mathrm{bA}$ \\
\hline
\end{tabular}

Fonte: Autores.

Para a camada de 0,10-0,20 m os tratamentos que tiveram decréscimo nos valores médios da DS foram o CS e o MP, respectivamente de $1,16 \mathrm{Mg} \mathrm{m}^{-3}$ para $1,01 \mathrm{Mg} \mathrm{m}^{-3}$ e de $1,17 \mathrm{Mg} \mathrm{m}^{-3}$ para 1,03 $\mathrm{Mg} \mathrm{m}^{-3}$ (Tabela 3). O fato da CS reduzir a DS se justifica devido a mesma apresentar raiz pivotante e profunda capaz de transpor as camadas compactadas, que ao se decomporem, favorecem as culturas subsequentes permitindo o desenvolvimento dos seus sistemas radiculares pelos bioporos produzidos. As melhorias proporcionadas pelas plantas de cobertura também foram observadas por Calegari et al. (1993).

Para a camada de 0,20-0,30m ao analisarmos os dados de DS, vemos que os tratamentos que propiciaram reduções

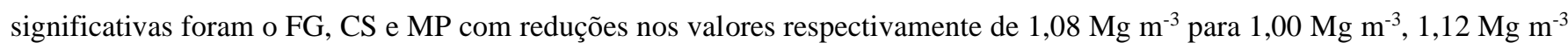
para $1,04 \mathrm{Mg} \mathrm{m}^{-3}$ e $1,07 \mathrm{Mg} \mathrm{m}^{-3}$ para $0,96 \mathrm{Mg} \mathrm{m}^{-3}$. 
Figura 1. Valores médios da densidade (A e B) e condutividade hidráulica do solo saturado do solo-Ksat (C e D), respectivamente antes da implantação (2018a) e após a colheita da cultura do milho (2018b), nas camadas de 0,00-0,10, 0,10$0,20,0,20-0,30 \mathrm{~m}$.

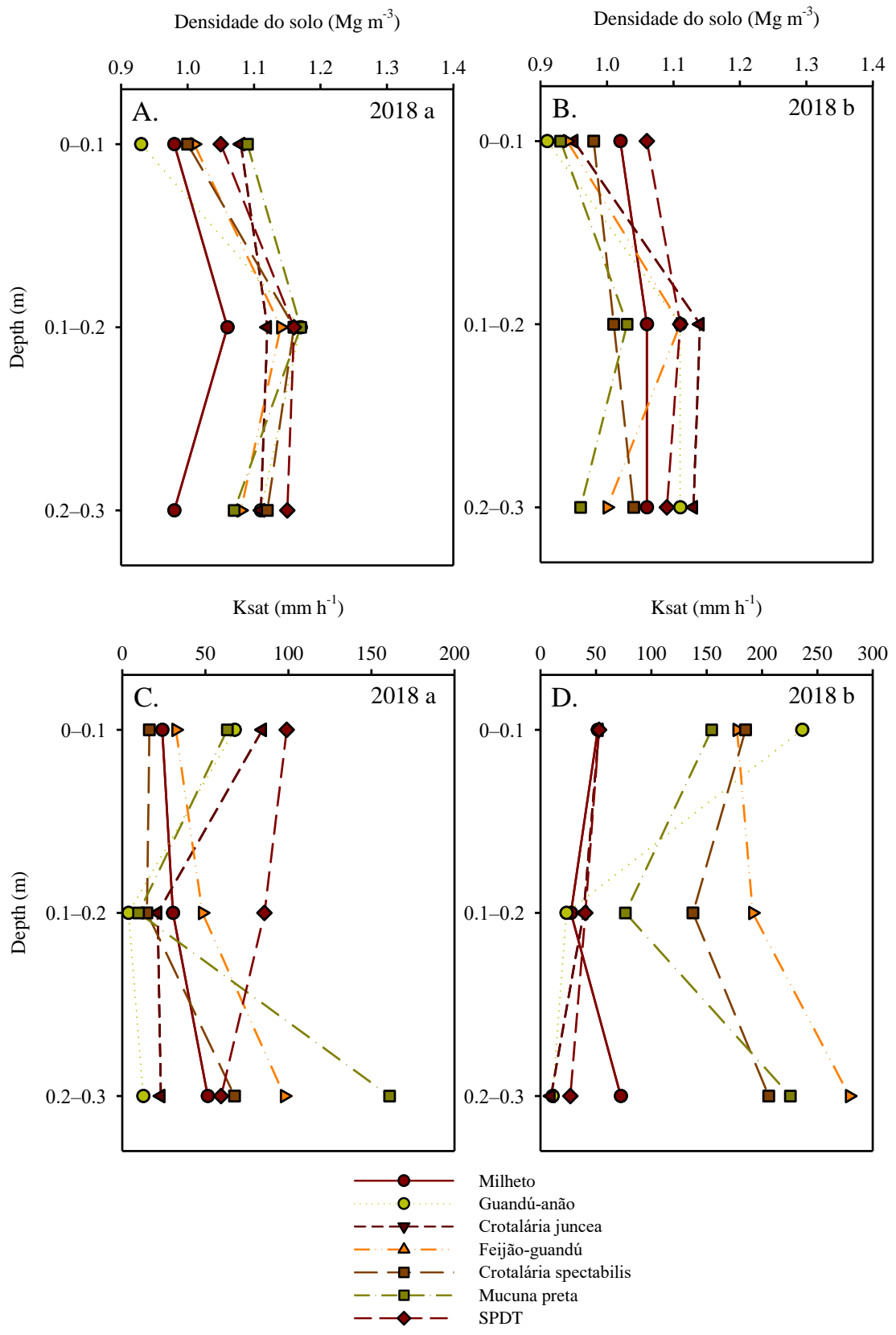

Fonte: Autores.

Quanto aos efeitos de tratamentos na época antes da implantação da cultura do milho (2018a) verifica-se que a DS do tratamento com MI $\left(0,98 \mathrm{Mg} \mathrm{m}^{-3}\right)$ foi o que apresentou o menor valor, contudo não diferiu ao FG $\left(1,08 \mathrm{Mg} \mathrm{m}^{-3}\right)$ e do MP (1,07 $\left.\mathrm{Mg} \mathrm{m}^{-3}\right)$, embora tenha apresentado diferença significativa dos tratamentos de $\mathrm{GA}\left(1,11 \mathrm{Mg} \mathrm{m}^{-3}\right), \mathrm{CJ}\left(1,11 \mathrm{Mg} \mathrm{m}^{-3}\right), \mathrm{CS}(1,12$ $\left.\mathrm{Mg} \mathrm{m}^{-3}\right)$ e $\operatorname{SPDT}\left(1,15 \mathrm{Mg} \mathrm{m}^{-3}\right)$.

Já na condição após a colheita da cultura do milho (2018d), pode-se observar que o tratamento com menor média da DS foi o MP (0,96 $\left.\mathrm{Mg} \mathrm{m}^{-3}\right)$, não diferindo do MI (1,06 $\left.\mathrm{Mg} \mathrm{m}^{-3}\right)$, FG (1,00 $\left.\mathrm{Mg} \mathrm{m}^{-3}\right)$ e CS (1,04 Mg m $\left.\mathrm{m}^{-3}\right)$, diferindo-se dos tratamentos de GA $\left(1,11 \mathrm{Mg} \mathrm{m}^{-3}\right)$, CJ $\left(1,13 \mathrm{Mg} \mathrm{m}^{-3}\right)$ e SPDT $\left(1,09 \mathrm{Mg} \mathrm{m}^{-3}\right)$, evidenciando desta forma, que após a senescência das raízes, os bioporos deixados pelas diferentes plantas de cobertura apresentaram efeitos positivos na redução da DS para a 
cultura sucessora.

Os baixos valores de DS na camada de 0,00-0,10m são explicados devido ao maior acúmulo de matéria orgânica, bem como revolvimento pelos mecanismos da semeadora no momento da semeadura das culturas. Desta forma pode-se observar que a média geral dos tratamentos tem menor valor de densidade nesta camada (Tabela 3, Figura 1).

Verifica-se que a camada de 0,00-0,10m apresentou valor médio de $1,0 \mathrm{Mg} \mathrm{m}^{-3}$ de densidade e a camada de 0,10 $0,20 \mathrm{~m}$ apresenta o maior valor com $1,11 \mathrm{Mg} \mathrm{m}^{-3}$. Estando os valores abaixo dos valores considerados críticos, que segundo Reichert et al. (2009) para solos argilosos é de $1,35 \mathrm{Mg} \mathrm{m}^{-3}$ ou superior.

Quanto ao Ksat pode-se constatar na camada 0,0-0,1m que os tratamentos de GA, FG e CS foram os que proporcionaram aumento dos valores respectivamente de $67,64 \mathrm{~mm} \mathrm{~h}^{-1}$ para $236,19 \mathrm{~mm} \mathrm{~h}^{-1} ; 32,22 \mathrm{~mm} \mathrm{~h}^{-1}$ para $177,30 \mathrm{~mm} \mathrm{~h}^{-1}$ e 16,24 $\mathrm{mm} \mathrm{h}^{-1}$ para $185,04 \mathrm{~mm} \mathrm{~h}^{-1}$. Nos períodos antes e depois do milho, constata-se nesta camada, que a ksat do tratamento MI (176,56 $\left.\mathrm{mm} \mathrm{h}^{-1}\right)$ foi o que apresentou o maior valor, não diferindo do tratamento de SPDT $\left(85,48 \mathrm{~mm} \mathrm{~h}^{-1}\right)$, diferindo-se dos tratamentos de GA $\left(3,68 \mathrm{~mm} \mathrm{~h}^{-1}\right)$, CJ $\left(21,22 \mathrm{~mm} \mathrm{~h}^{-1}\right)$, FG (48,17 $\left.\mathrm{mm} \mathrm{h}^{-1}\right)$, CS (14,78 $\left.\mathrm{mm} \mathrm{h}^{-1}\right)$ e MP (9,69 mm h-1) (Tabela 3).

Já no período após a colheita do milho, pode-se observar que o tratamento que apresentou o maior valor de ksat foi o FG $\left(191,45 \mathrm{~mm} \mathrm{~h}^{-1}\right)$, porém não diferiu dos tratamentos de CS $\left(137,59 \mathrm{~mm} \mathrm{~h}^{-1}\right)$ e MP $\left(76,51 \mathrm{~mm} \mathrm{~h}^{-1}\right)$, contudo diferiu para os demais tratamentos de MI $\left(27,41 \mathrm{~mm} \mathrm{~h}^{-1}\right)$, GA $\left(23,29 \mathrm{~mm} \mathrm{~h}^{-1}\right)$, CJ $\left(38,73 \mathrm{~mm} \mathrm{~h}^{-1}\right)$ e SPDT $\left(40,31 \mathrm{~mm} \mathrm{~h}^{-1}\right)$.

Segundo Ruffato et al. (2019) nota-se que as espécies de coberturas de Guandu anão e Crotalaria spectabilis foram espécies que apresentaram bom potencial estruturante do solo. Sulzbach et al. (2017) corrobora também a estes resultados e ressalta que o guandú anão apresentou resultados positivos na reestruturação do solo.

Para o Ksat na camada 0,1-0,2m (2018d) nota-se que os tratamentos de FG e CS foram os que proporcionaram aumento dos valores, respectivamente de $48,17 \mathrm{~mm} \mathrm{~h}^{-1}$ para 191,45 $\mathrm{mm} \mathrm{h}^{-1}$, e 14,78 $\mathrm{mm} \mathrm{h}^{-1}$ para 137,59 $\mathrm{mm} \mathrm{h}^{-1}$ (Tabela3). Já na camada de 0,20-0,30 que o tratamento que proporcionou aumento foi o FG de 97,59 $\mathrm{mm} \mathrm{h}^{-1}$ para $278,90 \mathrm{~mm} \mathrm{~h}^{-1}$. Sendo este o maior valor, não diferenciou significativamente dos tratamentos de MI $\left(72,37 \mathrm{~mm} \mathrm{~h}^{-1}\right)$, CS $\left(205,96 \mathrm{~mm} \mathrm{~h}^{-1}\right)$ e MP $(225,44$ $\mathrm{mm} \mathrm{h}^{-1}$ ), diferindo dos demais tratamentos.

Outro aspecto a observar quanto à Kat são os elevados valores do coeficiente de variação (CV), acima de 90\%. Isso se deve ao fato de que até mesmo a menor mudança na estrutura do solo, implica diretamente em mudança deste parâmetro. Estes elevados valores de CV's corroboram aos encontrados por Mesquita e Moraes (2004) os quais foram superiores a $70 \%$. Segundo Eguchi et al. (2003), o elevado CV dos dados pode ser esclarecido pela concentração de raízes, heterogeneidade estrutural do solo em estudo, atividade microbiológica, composição do solo, dentre outros fatores, podendo ser constatado pela camada de 0,00-0,10 m, que ressalva maior concentração de raízes assim como revolvimento no momento da semeadura, refletindo por tanto no maior valor de média geral, para o coeficiente de variação.

Na Tabela 4 nota-se que os valores da porosidade total (PT) na camada de 0,00-0,10m sofreram aumento devido aos tratamentos CJ e MP, na comparação do antes e após o cultivo do milho, com incremento de 57,90\% para 62,82\% e de 57,56\% para 63,54\%, respectivamente. Quanto a diferença entre os tratamentos no momento anterior a implantação do milho, verificase que que o GA apresentou valor maior que os demais, no entanto não diferiu do MI, FG e CS. Já no momento posterior os tratamentos (2018d) o GA (64,35\%) e MP (63,54\%) apresentaram os valores mais elevados, porém não diferindo dos demais tratamentos. 
Tabela 4. Valores médios da porosidade total (PT), microporosidade (Micro) e macroporosidade (Macro) do solo nas camadas de 0,00-0,10, 0,10-0,20 e 0,20-0,30 m, antes da implantação (2018a) e após a colheita da cultura do milho (2018b). MI: milheto; GA: guandú-anão; CJ: crotalária juncea; FG: feijão-guandú; CS: crotalária spectabilis; MP: mucuna preta; SPDT: sistema plantio direto tradicional. Médias de tratamentos seguidas de mesmas letras, minúscula na coluna e maiúscula na linha, não diferem entre si pelo Teste de Tukey a 5\% de significância.

\begin{tabular}{|c|c|c|c|c|c|c|}
\hline Tratamentos & $\mathrm{PT}(\%)$ & & Micro $(\%)$ & & Macro (\%) & \\
\hline \multicolumn{7}{|c|}{ Camada de 0,00-0,10 m } \\
\hline & $2018 \mathrm{a}$ & $2018 d$ & $2018 \mathrm{a}$ & $2018 d$ & $2018 \mathrm{a}$ & $2018 d$ \\
\hline MI & $61,75 \mathrm{abA}$ & $60,30 \mathrm{abA}$ & $46,98 \mathrm{abA}$ & $46,54 \mathrm{abA}$ & $14,77 \mathrm{aA}$ & $13,76 \mathrm{aA}$ \\
\hline GA & $63,54 \mathrm{aA}$ & $64,35 \mathrm{aA}$ & $48,74 \mathrm{abA}$ & $48,19 \mathrm{abA}$ & $14,79 \mathrm{aA}$ & $16,17 \mathrm{aA}$ \\
\hline $\mathrm{CJ}$ & $57,90 \mathrm{bB}$ & $62,82 \mathrm{abA}$ & $41,33 \mathrm{bB}$ & $51,36 \mathrm{aA}$ & $16,56 \mathrm{aA}$ & $11,46 \mathrm{aB}$ \\
\hline $\mathrm{FG}$ & $60,73 \mathrm{abA}$ & $63,15 \mathrm{abA}$ & $49,47 \mathrm{aA}$ & $45,75 \mathrm{abA}$ & $11,26 \mathrm{aB}$ & $17,39 \mathrm{aA}$ \\
\hline $\mathrm{CS}$ & $61,19 \mathrm{abA}$ & $61,89 \mathrm{abA}$ & $48,34 \mathrm{abA}$ & $46,11 \mathrm{abA}$ & $12,85 \mathrm{aA}$ & $15,78 \mathrm{aA}$ \\
\hline MP & $57,56 \mathrm{bB}$ & $63,54 \mathrm{aA}$ & $44,82 \mathrm{abA}$ & $46,87 \mathrm{abA}$ & $12,74 \mathrm{aB}$ & $16,67 \mathrm{aA}$ \\
\hline SPDT & $59,22 \mathrm{abA}$ & $58,75 \mathrm{abA}$ & 44,07abA & $43,07 \mathrm{bA}$ & $15,15 \mathrm{aA}$ & $15,68 \mathrm{aA}$ \\
\hline \multicolumn{7}{|c|}{ Camada de $0,10-0,20 \mathrm{~m}$} \\
\hline MI & $59,02 \mathrm{aA}$ & $58,78 \mathrm{aA}$ & $42,34 \mathrm{aA}$ & $41,74 \mathrm{aA}$ & $16,68 \mathrm{aA}$ & $17,04 \mathrm{aA}$ \\
\hline GA & $54,86 \mathrm{aA}$ & $57,01 \mathrm{aA}$ & $38,54 \mathrm{aA}$ & $40,10 \mathrm{aA}$ & $16,31 \mathrm{abA}$ & $16,91 \mathrm{aA}$ \\
\hline $\mathrm{CJ}$ & $56,77 \mathrm{aA}$ & $56,03 \mathrm{aA}$ & $39,95 \mathrm{aA}$ & $40,58 \mathrm{aA}$ & $16,82 \mathrm{abA}$ & $15,44 \mathrm{aA}$ \\
\hline FG & $55,81 \mathrm{aA}$ & $57,19 \mathrm{aA}$ & $39,91 \mathrm{aA}$ & $42,61 \mathrm{aA}$ & $15,90 \mathrm{bA}$ & $14,58 \mathrm{aA}$ \\
\hline CS & $55,18 \mathrm{aB}$ & $61,18 \mathrm{aA}$ & $40,71 \mathrm{aA}$ & $43,83 \mathrm{aA}$ & $14,46 \mathrm{bA}$ & $17,34 \mathrm{aA}$ \\
\hline MP & $54,75 \mathrm{aB}$ & $60,17 \mathrm{aA}$ & $40,85 \mathrm{aA}$ & $42,07 \mathrm{aA}$ & $13,90 \mathrm{bA}$ & $18,10 \mathrm{aA}$ \\
\hline SPDT & $55,29 \mathrm{aA}$ & $57,00 \mathrm{aA}$ & $37,15 \mathrm{aB}$ & $41,08 \mathrm{aA}$ & $18,13 \mathrm{abA}$ & $15,92 \mathrm{aA}$ \\
\hline \multicolumn{7}{|c|}{ Camada de $0,20-0,30 \mathrm{~m}$} \\
\hline MI & $63,18 \mathrm{aA}$ & $60,26 a b c B$ & $46,45 \mathrm{aA}$ & $42,64 \mathrm{abB}$ & $16,73 \mathrm{abA}$ & $17,61 \mathrm{abcA}$ \\
\hline GA & $58,33 \mathrm{bA}$ & $58,44 \mathrm{bcA}$ & $42,00 \mathrm{abcA}$ & $42,80 \mathrm{abA}$ & $16,33 \mathrm{abA}$ & $15,64 b c A$ \\
\hline CJ & $58,50 \mathrm{bA}$ & $57,48 \mathrm{cA}$ & $40,86 \mathrm{bcA}$ & $42,62 \mathrm{abA}$ & $17,65 \mathrm{abA}$ & $14,85 \mathrm{cB}$ \\
\hline FG & $59,41 \mathrm{abB}$ & $62,47 \mathrm{abA}$ & $39,26 \mathrm{cA}$ & $41,87 \mathrm{abA}$ & $20,15 \mathrm{aA}$ & $20,60 \mathrm{aA}$ \\
\hline $\mathrm{CS}$ & $58,14 \mathrm{bB}$ & $60,93 \mathrm{abcdA}$ & $40,19 \mathrm{bcA}$ & $41,46 \mathrm{bA}$ & $17,95 \mathrm{abA}$ & $19,47 \mathrm{abA}$ \\
\hline MP & $60,0 \mathrm{abB}$ & 63,91aA & 44,60abA & $45,71 \mathrm{aA}$ & $15,45 \mathrm{bB}$ & $18,20 \mathrm{abcA}$ \\
\hline SPDT & $56,93 \mathrm{bA}$ & $59,10 \mathrm{bcA}$ & $39,36 \mathrm{cA}$ & $40,69 \mathrm{bA}$ & $17,57 \mathrm{abA}$ & $18,41 \mathrm{abcA}$ \\
\hline
\end{tabular}

Fonte: Autores.

Na camada de 0,10-0,20m a PT dos tratamentos CS e a MP apresentaram comportamento crescente. Nos dois períodos avaliados individualmente não houve diferença significativa entre os tratamentos (Tabela 4).

Na camada de 0,20-0,30m a PT foi incrementada nos tratamentos, FG, CS e MP e redução no MI. No período antes da implantação, verificou-se que o tratamento MI, apresentou maior percentual, o qual diferiu dos tratamentos GA, CJ, CS, MP e SPDT. No período após a colheita do milho, percebe-se que o MP apresentou maior valor de PT e diferiu significativamente do GA, CJ e do SPDT. 
Figura 2. Valores médios da porosidade total-PT (A e B), microporosidade-Micro (C e D) e macroporosidade-Macro (E e F), respectivamente antes da implantação (2018a) e após a colheita da cultura do milho (2018b), nas camadas de 0,00-0,10, 0,10$0,20,0,20-0,30 \mathrm{~m}$.
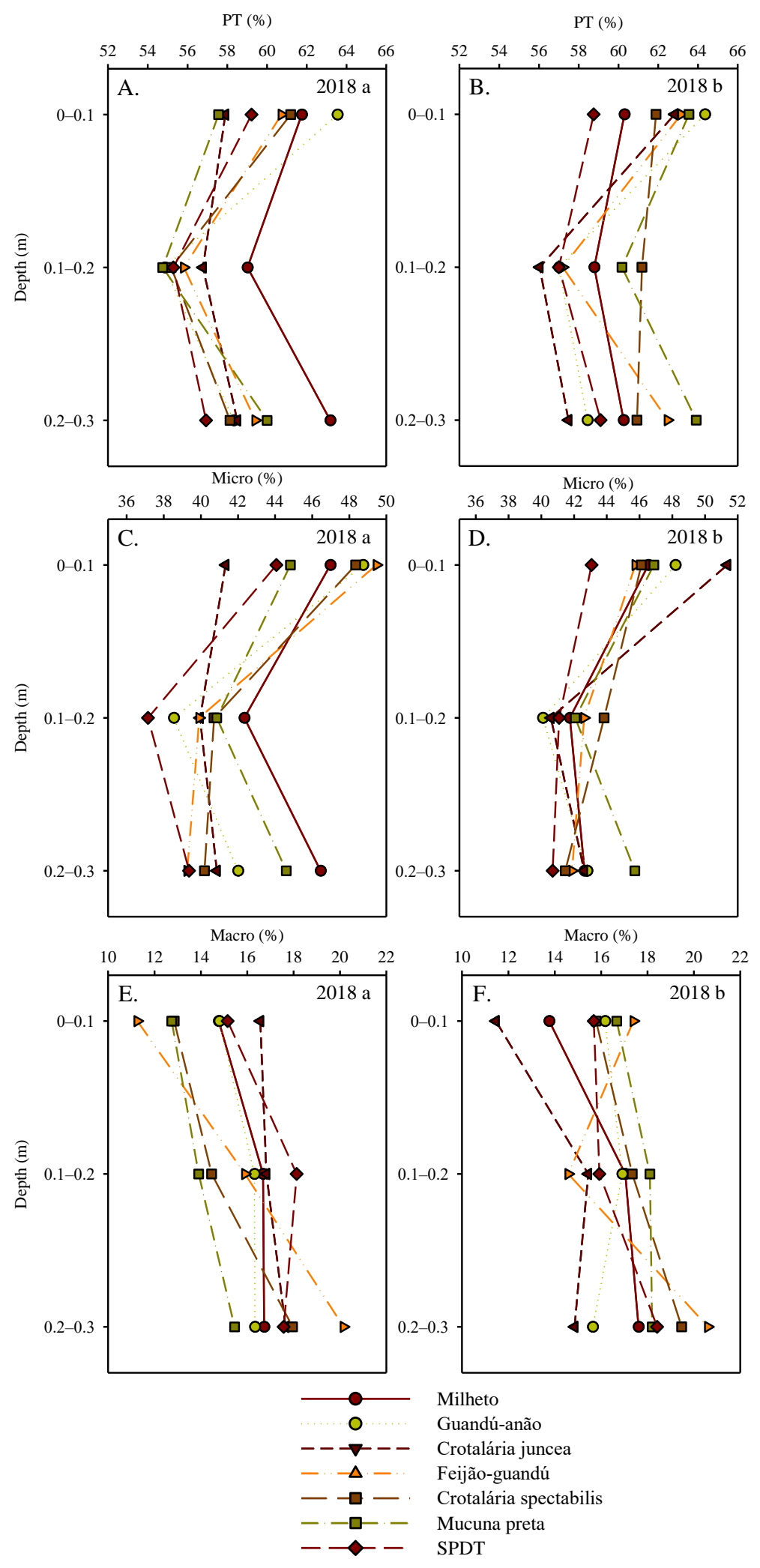

Fonte: Autores. 
Quanto a microporosidade, os tratamentos MI e o SPDT apresentaram incremento ao comparar as épocas (2018a x 2018d), embora não tenha ocorrido diferenças significativas entre os tratamentos nas duas épocas avaliadas. Antes da implantação da cultura do milho, o tratamento MI se destacou com o maior percentual da microporosidade do solo, diferindo dos tratamentos CJ, FG, CS e SPDT. Após a colheita do milho, o tratamento MP apresentou maior valor de microporosidade, diferindo dos tratamentos CS e SPDT.

As observações referentes a microporosidade mostram que o tratamento CJ apresentou aumento deste atributo na comparação do período antecedente e posterior ao cultivo do milho. Antes do cultivo do milho, na camada de 0,20-0,30, o tratamento que apresentou o maior valor foi o MI (46,45\%), não diferindo dos tratamentos de GA (42,00\%) e MP (44,60\%), e diferindo-se dos tratamentos de CJ (40,86\%), FG (39,26\%), CS (40,19\%) e SPDT $(39,36 \%)$. Depois do cultivo, constata-se que o tratamento que apresentou o maior valor foi a MP (45,71\%), não diferindo do MI (42,64\%), GA (42,80\%), CJ (42,62\%), FG (41,87\%), e diferindo-se da CS (41,46\%) e SPDT (40,69\%).

Para a macroporosidade na camada 0-0,1m, o MP aumentou o percentual entre os períodos avaliados (2018a x 2018d) e o CJ reduziu. No primeiro período avaliado o tratamento FG apresentou maior valor de macroporosidade, diferindo significativamente do MP. Já no segundo instante o FG se destacou, diferindo apenas dos tratamentos GA e CJ.

A macroporosidade, embora não tenha ocorrido diferença significativa entre os períodos avaliados, os tratamentos CS e MP apresentaram tendência de aumento. Isso pode ser explicado pelo fato destes tratamentos propiciarem redução da densidade do solo nesta camada (Tabela 3).

Já a macroporosidade na camada 0,2-0,3m, os tratamentos FG e MP aumentaram seu percentual na comparação entre os momentos. O tratamento que apresentou o maior valor antes do milho foi o FG (20,15\%), não diferindo dos tratamentos MI (16,73\%), GA (16,33\%), CJ (17,65\%), CS (17,95\%) e SPDT (17,57\%), diferindo-se do tratamento de MP (15,45\%). Estes valores demonstram as boas condições estruturais da área experimental pois todos os valores de macroporosidade estão acima do valor considerado crítico de $10 \%$ Reynolds et al. (2002).

A Figura 2 evidencia melhor a tendência de incremento da macroporosidade na camada 0,1-0,2m, em especial nos tratamentos CS e MP. De modo geral verificou-se que com incremento na DS ocorreu redução na PT e na macroporosidade em todas as camadas avaliadas (Tabela 3 x Tabela 4), o que já era esperado. A média geral da PT na camada de 0,00-0,10m foi a que apresentou o maior valor $(61,19 \%)$ e a camada de $0,10-0,20 \mathrm{~m}$ foi a que apresentou o menor valor de PT (57,07\%) (Figura 2). Estes valores de PT são superiores ao valor de 50\%, considerado ideal de acordo com Andrade e Stone (2009). ASSIS et al., 2009, também afirmam que o solo fisicamente ideal é aquele que apresenta PT de 50\%, cerca de 17\% de macroporos e de $33 \%$ de microporos. Assim, os valores encontrados destes atributos neste trabalho evidenciam o bom estado estrutural do solo da área experimental.

Na Tabela 5 pode-se observar que não houve diferença significativa quanto ao rendimento de grãos de milho. Estes dados, corroboram aos encontrados por Ruffato et al. (2019) e Sulzbach et al. (2017) que ressaltam que o rendimento de grãos de soja não apresentou diferenças significativas entre as espécies de cobertura avaliadas. $O$ fato de não haver diferença significativa entre os tratamentos quanto ao rendimento de grãos de milho pode estar atrelado ao solo ter apresentado boa qualidade estrutural, conforme observado neste trabalho. 
Tabela 5. Rendimentos dos grãos de milho em função dos tratamentos envolvendo espécies de cobertura de verão (valores médios de três repetições). Médias de tratamentos seguidas de mesmas letras na coluna, não diferem entre si pelo Teste de Tukey a 5\% de significância. MI: milheto; GA: guandú-anão; CJ: crotalária juncea; FG: feijão-guandú; CS: crotalária spectabilis; MP: mucuna preta; SPDT: sistema plantio direto tradicional.

\begin{tabular}{lc}
\hline Tratamento & Rendimento dos grãos $\left(\mathrm{kg} \mathrm{ha}^{-1}\right)$ \\
\hline MI & $6825 \mathrm{a}$ \\
GA & $6467 \mathrm{a}$ \\
CJ & $6365 \mathrm{a}$ \\
FG & $6160 \mathrm{a}$ \\
CS & $6295 \mathrm{a}$ \\
MP & $5790 \mathrm{a}$ \\
SPDT & $6487 \mathrm{a}$ \\
\hline
\end{tabular}

Fonte: Autores.

\section{Conclusão}

As espécies de cobertura que apresentaram maior eficiência na reestruturação do solo foram a crotalária spectábilis e a mucuna preta.

Não houve diferença significativa quanto ao rendimento de grãos de milho, evidenciando as boas condições estruturais do solo da área experimental.

\section{Referências}

Álvarez, C. R., Torres Duggan, M., Chamorro, E. R., \& Taboada, M. A. (2009). Descompactación de suelos franco limosos en siembra directa: efectos sobre las propiedades edáficas y los cultivos. Ciencia del suelo, 27(2), 159-169.

Andrade, R. D. S., \& Stone, L. F. (2009). Índice S como indicador da qualidade física de solos do cerrado brasileiro. Revista Brasileira de Engenharia Agrícola e Ambiental, 13(4), 382-388.

Assis, R. L. D., Lazarini, G. D., Lanças, K. P., \& Cargnelutti Filho, A. (2009). Avaliação da resistência do solo à penetração em diferentes solos com a variação do teor de água. Engenharia Agrícola, 29, 558-568.

Bareta Junior, E, da Silva, A. A. P., Sens, T. M. Z. G., Colecha, K., Rampim, L., \& Pott, C. A. (2021). Soil physical properties in variable levels of soil compaction. Research, Society and Development, 10(2), e2341028686-e2341028686.

Calegari, A., Mondardo, A., Bulisani, E. A., Wildner, L. D. P., da Costa, M. B. B., Alcântara, P. B., \& Amado, T. J. C. (1993). Adubação verde no sul do Brasil (2a edição), 1993, 346 p.

Carvalho, A. D., Bustamante, M. D. C., Alcântara, F. D., Resck, I. S., \& Lemos, S. S. (2009). Characterization by solid-state CPMAS 13C NMR spectroscopy of decomposing plant residues in conventional and no-tillage systems in Central Brazil. Soil and Tillage Research, 102(1), 144-150.

CONAB - Companhia Nacional de Abastecimento. Acompanhamento da safra Brasileira, v.5-safra 2017/2018-n.6. Sexto levantamento, março 2018.

Eguchi, E. S., Silva, E. D., \& Oliveira, M. D. (2003). Variabilidade espacial da condutividade hidráulica do solo saturado e da taxa de infiltração básica determinadas "in situ". Ciência e Agrotecnologia, 1607-1613.

Melo Filho, J. F. D., Carvalho, L. L. D., Silveira, D. D. C., Sacramento, J. A. A. S. D., \& Silveira, E. C. P. (2009). Índice de qualidade em um Latossolo Amarelo coeso cultivado com citros. Revista Brasileira de Fruticultura, 31, 1168-1177.

Mesquita, M. D. G. B. D. F., \& Moraes, S. O. (2004). A dependência entre a condutividade hidráulica saturada e atributos físicos do solo. Ciência Rural, 34(3), 963-969.

Panachuki, E., Bertol, I., Alves Sobrinho, T., Oliveira, P. T. S. D., \& Rodrigues, D. B. B. (2011). Soil and water loss and water infiltration in red latosol under different management systems. Revista Brasileira de Ciência do Solo, 35(5), 1777-1786.

Portugal, J. R., Arf, O., Peres, A. R., Gitti, D. D. C., \& Garcia, N. F. S. (2017). Coberturas vegetais, doses de nitrogênio e inoculação com Azospirillum brasilense em milho no Cerrado. Revista Ciência Agronômica, 48, 639-649.

Reichert, J. M., Suzuki, L. E. A. S., Reinert, D. J., Horn, R., \& Håkansson, I. (2009). Reference bulk density and critical degree-of-compactness for no-till crop production in subtropical highly weathered soils. Soil and Tillage Research, 102(2), 242-254. 
Research, Society and Development, v. 10, n. 16, e220101623786, 2021

(CC BY 4.0) | ISSN 2525-3409 | DOI: http://dx.doi.org/10.33448/rsd-v10i16.23786

Reynolds, W. D., Bowman, B. T., Drury, C. F., Tan, C. S., \& Lu, X. (2002). Indicators of good soil physical quality: density and storage parameters. Geoderma, 110(1-2), 131-146.

Ruffato, G. G., Secco, D., Zanão Junior, L. A., Tokura, L. K., de Marins, A. C., Villa, B. D., \& do Nascimento, L. F. J. (2019). Structuring of a haplortox by cover crops and their effects on the yield of soybean grains. Journal of Agricultural Science, 11(5), 309-313.

Sulzbach, L. G., Secco, D., Tokura, L. K., de Villa, B., \& Ruffato, G. G. (2017). Implicações de espécies de cobertura em parâmetros físico-hídricos de um latossolo argiloso e no rendimento de grãos de soja. Acta Iguazu, 6(5), 280-286.

Spliethoff, J., Pott, C. A., Rampim, L., Watzlawick, L. F., \& Jadoski, S. O. (2020). Limites de compactação do solo para Ilex paraguariensis. Research, Society and Development, 9(5), e23953101-e23953101.

Tisdall, J. M., \& Oades, J. M. (1982). Organic matter and water-stable aggregates in soils. Journal of soil science, 33(2), 141-163.

Tokura, L. K., Secco, D., Júnior, L. A. Z., Siqueira, J. A. C., de Souza, S. N. M., Baricatti, R. A., \& Center, N. M. R. (2018). Structuring of a Haplortox by soil cover species. Journal of Agricultural Science, 10(11), 299-310.

Tokura, L. K., Secco, D., Júnior, L. A. Z., Siqueira, J. A. C., Alovisi, A. M. T., Barison, A., \& Zin, Z. (2021). Use of cover crops in Oxisol and its effects on yield and soybean oil content. Research, Society and Development, 10(12), e353101220514-e353101220514.

Vieira, J. M., Romero, R. E., Ferreira, T. O., \& Assis Júnior, R. N. D. (2012). Contribuição de material amorfo na gênese de horizontes coesos em Argissolos dos Tabuleiros Costeiros do Ceará. Revista Ciência Agronômica, 43, 623-632.

Warrick, A. W. (1980). Spatial variability of soil physical properties in the field. Application of soil physics., 319-344.

Zin, Z., Secco, D., Villa, B. D., Tokura, L. K., Alovisi, A. M. T., de Marins, A. C., \& Silveira, L. D. (2019). Nutrient cycling by cover species and yield of soybean grains in a clayey Oxisol under no-tillage system. Journal of Agricultural Science, 11(7), 294-302. 